\title{
Temporal patterns of soluble adhesion molecules in cerebrospinal fluid and plasma in patients with the acute brain infraction
}

\author{
Vesna Selakovic ${ }^{\mathrm{a}}$, Ranko Raicevic ${ }^{\mathrm{b}}$ and Lidija Radenovic ${ }^{\mathrm{c}, *}$ \\ ${ }^{\mathrm{a}}$ Institute for Medical Research, MMA, Belgrade, Serbia \\ ${ }^{\mathrm{b}}$ Clinic of Neurology, MMA, Belgrade, Serbia \\ ${ }^{\mathrm{c}}$ Department of Physiology and Biochemistry, Faculty of Biology, University of Belgrade, Serbia
}

\begin{abstract}
The aim of this study was to define concentration changes of soluble adhesion molecules (sICAM-1, sVCAM-1 and sE-Selectin) in cerebrospinal fluid and plasma, as well as, number of peripheral blood leukocytes and the albumin coefficient in the patients with the acute brain infarction. We also, analyzed the correlation between the measured levels, the infarct volume and the degree of neurological and the functional deficit. The study included 50 patients with the acute cerebral infarction and the control group consisted of 16 patients, age and sex matched. Obtained results showed significant increase in number of leukocytes, the albumin coefficient and the level of soluble adhesion molecules within the first seven days in patients. The highest values of measured parameters were noted within the third and the fourth day after the insult, which is the suggested period of maximal intensity of inflammatory reactions. Significant correlation was found between measured parameters and the infarct volume, the degree of neurological and the functional deficit. The results suggest that investigated parameters in CSF and blood represent a dynamic index of inflammatory events as one of the fundametal mechanisms responsible for neuron damage during acute phase of brain infarction.
\end{abstract}

Keywords: Brain infarction, inflammation, soluble adhesion molecules, leukocytes number, albumin coefficient, cerebrospinal fluid

\section{Introduction}

Inflammatory mechanisms play an important role in the risk of stroke and during the acute phase of brain ischemia, which contributes to functional outcome of patients. Systemic inflammatory mechanisms are associated with higher risk of stroke and vascular recurrences after a first ischemic stroke. Several inflammatory molecules are implicated during the acute phase of ischemic stroke, such as cytokines and adhesion cell molecules. Inflammatory cytokines and adhesion

*Corresponding author: Lidija Radenovic, Ph.D. Associate Professor, Department of Physiology and Biochemistry, Faculty of Biology, University of Belgrade, P.O.B. 52, Studentski trg 16, 11000 Belgrade, Serbia. Tel.: +381 11 3032356; Fax: +381 11 2638500; 3032356; E-mail: lidijar@bio.bg.ac.rs. molecules are related to the presence of early neurological deterioration and infarct volume [34].

Inflammation is important part in pathogenesis of acute brain ischemia [12,22-25]. Experimental studies showed that peripheral blood leukocytes migrate into the brain parenchyma within the first hours after ischemia $[1,11,13,17]$. Leukocytes contribute to the development of ischemic brain injury by causing capillary plugging, reducing cerebromicrovascular blood flow, increasing microvascular permeability, and secreting a different toxical mediators including reactive oxygen species, leukotrienes, cytokines, chemokines and cytotoxical enzymes which expanded the existing damage to surrounding tissue $[16,27,33,40]$.

Peripheral blood leukocytes migration requires prior adhesion to cerebral endothelial cells. This process is mediated by adhesion molecules on the sur- 
face of cerebral endothelial cells and leukocytes [23, 30]. During the acute cerebral ischemia three different adhesion molecules have emerged as key players in leukocytes-endothelial interactions: intercellular adhesion molecule 1 (ICAM-1) responsible for adhesion of mononuclear cells and granulocytes; vascular cell adhesion molecule 1 (VCAM-1) predominantly mediated adhesion of monocytes; and endothelial leukocyte adhesion molecule (E-Selectin) expressed at endothelial cells and facilitated adhesion of monocytes and granulocytes $[1,3,19,20,23,30]$. Under physiological conditions, adhesion molecules are scarcely expressed on the cell surface. Different pathophysiological processes might induce their expression [19,20,23]. Circulating, soluble forms of the adhesion molecules are formed as proteolytic release of their extracellular domains $[3,20$, 30]. The role of these soluble adhesion molecules in the acute cerebral ischemia in humans is not completely understood. Clinical data on adhesion molecule responses in cerebral ischemia are limited when compared with experimental studies. Longitudinal studies in human cerebrospinal fluid (CSF) and peripheral blood are rare. Inflammatory adhesion molecules play a key role in the development of ischemic lesions. Elevated plasma concentrations of soluble adhesion molecules are reported in stroke patients, but data are still controversial.

The aim of this study was to define time-dependent concentration changes of soluble adhesion molecules (sICAM-1, sVCAM-1 and sE-Selectin) in CSF and plasma, as well as, the number of peripheral blood leukocytes and albumin coefficient in the patients with the acute brain infarction. We also, analyzed the correlation between the measured levels, the infarct volume, and the degree of neurological and the functional deficit. Practical contribute of these investigations is that relatively simple determination of biochemical parameters in body fluids could help in early diagnosis of ischemic insult and prognosis of its outcome.

\section{Methods}

\subsection{Patients}

The study included 50 patients, 28 men and 22 woman, mean age $64 \pm 7$ years with the acute brain infarction. All patients had their first ever stroke and had a history of hypertension, hypercholesterolemia and diabetes, but without pre-treatment (such as statins or antiplatelet drugs) that can influence in the expression of inflammatory markers. The procedures followed were in accordance with the Helsinki Declaration of 1975 , also, after ethical committee and institutional approval, an informed consent was obtained from all patients before study entry. The diagnosis was established based on medical history, clinical examination, and cerebral CT scans in each single patient. In all the patients, a detailed neurological examination was performed at the hospitalization and every day during the first seven days after the onset of symptoms. Patients with presence of infections, other cerebral, inflammatory, pulmonary, hepatic, renal and malignant diseases, or immunosuppressive treatment, were excluded.

Cerebral CT scan was performed in all patients between 5th and 7th day after the onset of symptoms. Infarction volume was expressed by the largest diameter of hypodense area $(\mathrm{cm})$. The degrees of neurological and functional deficit during the acute phase were evaluated by appropriate neurological scales: Canadian neurological scale and Barthel index [7,36]. Canadian neurological scale measures level of consciousness, aphasia, orientation, facial paresis and power in arm, hand and leg on score from 1.5 (maximum deficit) to 10 (absence of deficit). The functional impairment was objectified by the Barthel index indicating the everyday ability of self-supply (score range between 0 , i.e. complete dependence for activities of daily life, and 20, i.e. no disability).

This evaluation was performed immediately after the blood and CSF samples were obtained. The degree of clinical expression of the brain infarction was determined by the Grotta scale - scale of clinical evaluation severeness of brain infraction (Mild, Moderate, Severe) [18]. Base on that, the study included 14 patients with mild, 21 with moderate and 15 patients with severe brain infarction.

The control group included 16 patients age and sex matched, subjected to diagnostic lumbar radiculography after the sudden onset of motor deficiency. Only patients without obstruction in the CSF passage and without history and clinical examination evidence of current inflammatory, renal, hepatic, pulmonary, malignant or psychiatric diseases and therapy were included in the study.

Soluble adhesion molecules concentration, number of leukocytes and albumin coefficient in patients with brain infarction were measured within the first seven days. The blood and CSF samples were obtained at 1-2 days (16 patients), 3-4 days (19 patients), and 5-7 days (15 patients) after the onset of infarction symptoms, according to admission at the Neurological Clinic. Patients in each group had comparable basal char- 
acteristics. First group (1-2 days) was consist of 4 patients with mild, 7 patients with moderate, and 5 patients with sever brain infarction. Second group (3-4 days) was consist of 5 patients with mild, 9 patients with moderate, and 5 patients with sever brain infarction. Third group (5-7 days) was consist of 5 patients with mild, 5 patients with moderate, and 5 patients with sever brain infarction. So, according to infarct severity (mild, moderate and severe) each temporal group of patients included uniform distribution.

\subsection{Biochemical analysis}

The blood and CSF samples were obtained at 9 AM from the patients (before the administration of any therapy). Peripheral blood samples were collected by venepuncture in heparinized tubes and stored on ice $\left(+4^{\circ} \mathrm{C}\right)$. CSF samples were collected during the lumbar puncture into the test tubes and held on ice $\left(+4{ }^{\circ} \mathrm{C}\right)$. Within $1 \mathrm{~h}$ of collection the simples were centrifuged at $3000 \mathrm{rpm}$ for 15 minutes at $+4{ }^{\circ} \mathrm{C}$. Plasma and CSF samples were stored at $-70^{\circ} \mathrm{C}$ until the biochemical analyses. The number of peripheral blood leukocytes was determined by hematological autoanalyzer. Albumin in CSF and plasma was measured by standard nephelometric method. The albumin coefficient (AC) was expressed as a ratio of albumin concentration in CSF and plasma, and was used to quantify the bloodbrain barrier damage.

\subsection{ELISA tests}

Concentrations of soluble adhesion molecules sICAM-1, sVCAM-1 and sE-Selectin were determined in plasma and CSF by ELISA-tests (R\&D Systems Europe, Abingdon, UK). Plasma samples were diluted with commercial diluents for detecting sICAM-1 in ratio $1: 80$, for s-VCAM-1 and sE-Selectin in ratio 1:50 and all CSF samples in ratio 1:1. The ELISA kits provide wells microplate coated with a monoclonal antibody to specific human adhesion molecules. Briefly, dilute samples were applied to wells and incubated with the respective horseradish peroxides-conjugated antiadhesion molecules antibody diluted with the conjugate diluents. After incubation within $90 \mathrm{~min}$ at room temperature, the microplates were washed five times with base buffer. Color was developed by the addition of substrate solution (tetramethyl-benzidine). The reaction was stopped after $30 \mathrm{~min}$ by addition sulfuric acid. The optical density of the developed color was measured at $450 \mathrm{~nm}$ with a correction at $620 \mathrm{~nm}$ us- ing a microplate reader (ELISA Processor II, Behring). The concentrations of soluble adhesion molecules in CSF and plasma samples (ng/ml) were calculated using a standard curve generated from absorbance values of increasing concentration of standards.

\subsection{Evaluation and statistical analyses}

Each assay was performed twice under identical conditions. Data are expressed as means \pm SD. The statistical significance of differences between groups was assessed by Student's $t$-test and by regression correlation analyses for the determination of the connection between the analyzed parameters and the degree of neurological deficit. A level of $p<0.05$ was considered statistically significant and $p<0.01$ as very significant.

\section{Results}

\subsection{Temporal patterns of peripheral blood leukocytes number and the albumin coefficient in patients with brain infraction and their values in different infarct severity}

The number of peripheral blood leukocytes in patients with the brain infarction (BI) during the first seven days after the ischemia onset was statistically significant increased compared to the control value (Table 1). Maximal increase in leukocytes number was obtained within the third and the fourth day after the insult (Table 1). The increase correlated to the disease severity and maximal value was found in patients with severe cerebral infarction (Table 2).

The albumin coefficient was expressed as a ratio of albumin concentration in CSF and plasma in the BI patients, and was used to quantify the blood-brain barrier damage. The albumin coefficient in the BI patients, during the first seven days after the insult was significantly increased compared to control (Table 1). Maximal albumin coefficient value was determined, again, within the third and the fourth day after the insult (Table 1). The increase of albumin coefficient correlated to the disease severity and maximal value was found in patients with severe cerebral infarction (Table 2). 
Table 1

A - number of peripheral blood leukocytes and albumin coefficient (AC), B - sICAM-1, sVCAM-1 and sE-Selectin concentrations in plasma and CSF in patients with brain infarction. Values are presented as mean value $\pm \mathrm{SD} .{ }^{*} p<0.05$ and ${ }^{* *} p<0.01$ represent statistically significant difference compared to control

\begin{tabular}{|c|c|c|c|c|c|}
\hline \multicolumn{6}{|c|}{ A } \\
\hline & & Control & $1-2$ days & $3-4$ days & 5-7 days \\
\hline & Leukocyte number $(\times 109 / \mathrm{L})$ & $5.4 \pm 0.8$ & $9.5 \pm 1.7^{* *}$ & $10.9 \pm 1.8^{* *}$ & $10.5 \pm 1.4^{* *}$ \\
\hline & $\mathrm{AC}$ & $4.6 \pm 1.3$ & $8.9 \pm 1.4^{* *}$ & $11.9 \pm 1.8^{* *}$ & $9.3 \pm 1.6^{* *}$ \\
\hline \multicolumn{6}{|c|}{ B } \\
\hline & & Control & $1-2$ days & $3-4$ days & 5-7 days \\
\hline \multirow[t]{2}{*}{ sICAM-1 (ng/ml) } & Plasma & $508.8 \pm 32.7$ & $669.4 \pm 75.3^{* *}$ & $730.6 \pm 68.1^{* *}$ & $695.1 \pm 43.8^{* *}$ \\
\hline & CSF & $2.16 \pm 0.69$ & $2.9 \pm 0.43^{* *}$ & $5.43 \pm 0.97^{* *}$ & $3.82 \pm 0.51^{* *}$ \\
\hline \multirow[t]{2}{*}{ sVCAM-1 (ng/ml) } & Plasma & $530.5 \pm 67.8$ & $658.0 \pm 91.9^{* *}$ & $1011.1 \pm 83.3^{* *}$ & $729.0 \pm 99.6^{* *}$ \\
\hline & $\mathrm{CSF}$ & $5.24 \pm 0.84$ & $8.42 \pm 1.58^{* *}$ & $17.89 \pm 1.78^{* *}$ & $10.32 \pm 1.32^{* *}$ \\
\hline \multirow[t]{2}{*}{ sE-Selectin (ng/ml) } & Plasma & $28.76 \pm 3.87$ & $53.7 \pm 10.02^{* *}$ & $57.25 \pm 8.7^{* *}$ & $36.4 \pm 5.29^{* *}$ \\
\hline & $\mathrm{CSF}$ & $0.11 \pm 0.008$ & $0.14 \pm 0.01^{* *}$ & $0.18 \pm 0.03^{* *}$ & $0.13 \pm 0.01^{*}$ \\
\hline
\end{tabular}

Table 2

A - number of peripheral blood leukocytes and albumin coefficient (AC), B - sICAM-1, sVCAM-1 and sE-Selectin concentrations in plasma and CSF in patients with different degree of brain infarction. The degree of clinical expression of the brain infarction was determined by the Grotta scale (mild, moderate, severe). Values are presented as mean value $\pm \mathrm{SD} .{ }^{*} p<0.05$ and ${ }^{* *} p<0.01$ represent statistically significant difference compared to control

\begin{tabular}{|c|c|c|c|c|c|}
\hline \multicolumn{6}{|c|}{ A } \\
\hline & & Control & Mild infarct & Moderate infarct & Severe infarct \\
\hline & Leukocyte number $(\times 109 / \mathrm{L})$ & $5.4 \pm 0.8$ & $8.5 \pm 0.9^{* *}$ & $10.8 \pm 1.5^{* *}$ & $11.3 \pm 1.5^{* *}$ \\
\hline & $\mathrm{AC}$ & $4.6 \pm 1.3$ & $8.6 \pm 1.3^{* *}$ & $9.71 \pm 1.6^{* *}$ & $12.2 \pm 1.8^{* *}$ \\
\hline \multicolumn{6}{|c|}{$\mathrm{B}$} \\
\hline & & Control & Mild infarct & Moderate infarct & Severe infarct \\
\hline \multirow[t]{2}{*}{ sVCAM-1 (ng/ml) } & Plasma & $530.5 \pm 67.8$ & $658.7 \pm 118.1^{* *}$ & $775.8 \pm 133.9^{* *}$ & $1013.6 \pm 91.9^{* *}$ \\
\hline & $\mathrm{CSF}$ & $5.24 \pm 0.84$ & $8.86 \pm 3.16^{* *}$ & $11.71 \pm 3.66^{* *}$ & $17.3 \pm 2.16^{* *}$ \\
\hline \multirow[t]{2}{*}{ sE-Selectin (ng/ml) } & Plasma & $28.76 \pm 3.87$ & $39.79 \pm 11.43^{* *}$ & $52.18 \pm 10.62^{* *}$ & $56.03 \pm 9.2^{* *}$ \\
\hline & CSF & $0.11 \pm 0.008$ & $0.13 \pm 0.01^{* *}$ & $0.15 \pm 0.01^{* *}$ & $0.18 \pm 0.03^{* *}$ \\
\hline
\end{tabular}

3.2. Temporal patterns of SICAM-1, sVCAM-1 and sE-Selectin plasma concentrations in patients with brain infraction and their values in different infarct severity

Plasma level of sICAM-1 in the BI patients, during the first seven days after the insult was significantly increased compared to control value (Table 1). Maximal sICAM-1 concentration was determined within the third and the fourth day after the insult (Table 1). Plasma level of sVCAM-1 in the BI patients, during the first seven days after the ischemia onset was significantly increased compared to control (Table 1). Maximal sVCAM-1 concentration was determined also, within the third and the fourth day after the insult (Table 1). Plasma concentration of sE-Selectin in the BI patients during the first seven days after the ischemia onset was, also, significantly increased compared to control value (Table 1). Maximal sE-Selectin concentration was determined, again, within the third and the fourth day after the insult (Table 1). The increase of sICAM-1, sVCAM-1, and sE-Selectin plasma levels correlated to the disease severity and maximal values were found in patients with severe cerebral infarction (Table 2).

\subsection{Temporal patterns of sICAM-1, sVCAM-1, and sE-Selectin concentrations in CSF in patients with brain infraction and their values in different infarct severity}

CSF concentration of sICAM-1 in the BI patients during the first seven days after the ischemia onset was significantly increased compared to control (Table 1). Maximal sICAM-1 level was obtained within the third and the fourth day after the insult (Table 1). CSF concentration of sVCAM-1 in the BI patients during the first seven days after the insult was significantly increased compared to control (Table 1). Maximal 

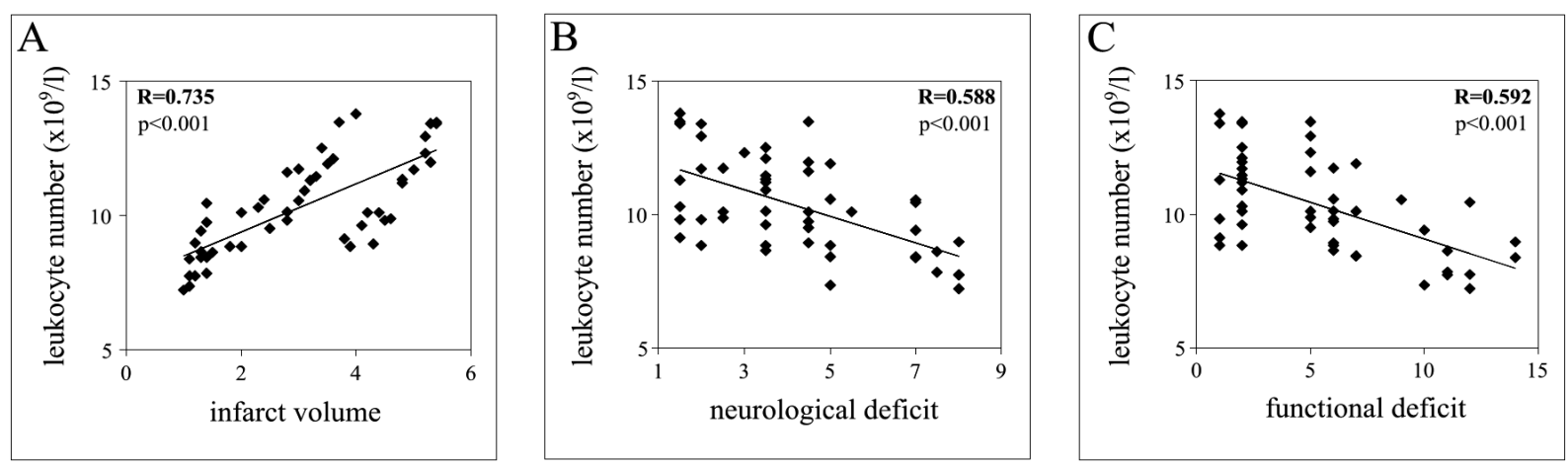

Fig. 1. Correlation between numbers of peripheral blood leukocytes with: A - the infarct volume, using CT scan (cm); B - the degree of neurological deficit, using Canadian scale (1.5-10); C - the degree of functional deficit, using Barthel index (0-20). R - correlation coefficient; $p<0.001-$ represents statistical significance.
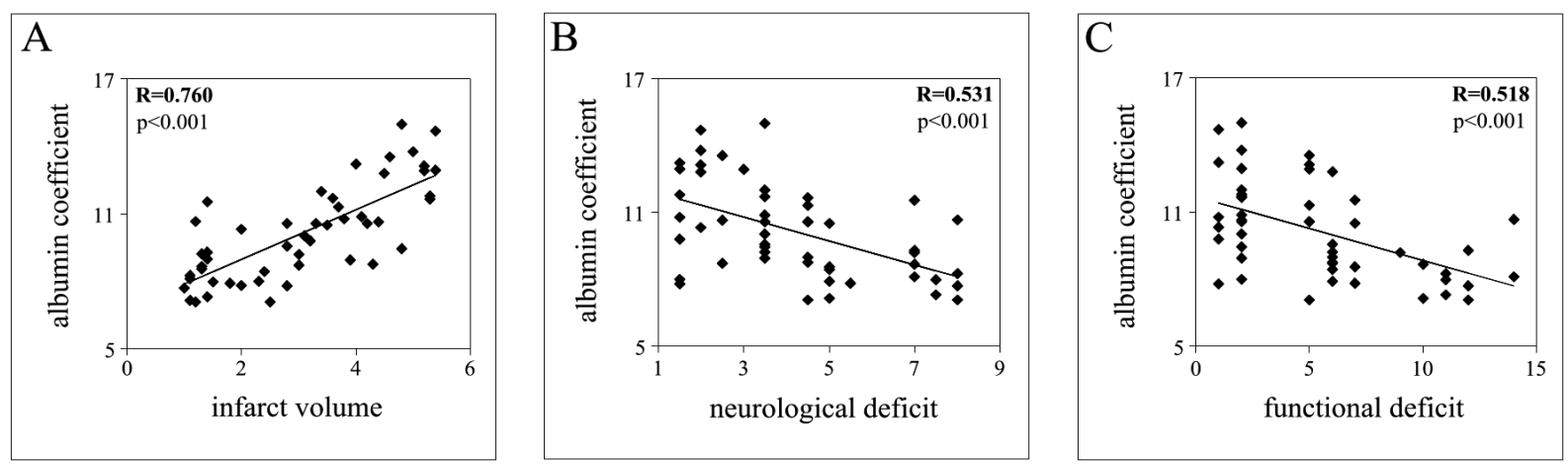

Fig. 2. Correlation between albumin coefficients with: A - the infarct volume, using CT scan ( $\mathrm{cm})$; B - the degree of neurological deficit, using Canadian scale (1.5-10); $\mathrm{C}-$ the degree of functional deficit, using Barthel index $(0-20) . \mathrm{R}-$ correlation coefficient; $p<0.001-$ represents statistical significance.

sVCAM-1 concentration was determined within the third and the fourth day after the insult (Table 1). CSF concentration of sE-Selectin in the BI patients during the first seven days after the ischemia onset was significantly increased compared to control value (Table 1). Maximal sE-Selectin level was obtained, again, within the third and the fourth day after the insult (Table 1). The increase of sICAM-1, sVCAM-1, and sE-Selectin CSF levels correlated to the disease severity and maximal values were found in patients with severe cerebral infarction (Table 2).

\subsection{Correlations between measured parameters and the infarct volume, the neurological and the functional deficit}

The correlation between measured parameters and the extent of infarct volume, the degree of neurological and functional deficit was determined by linear regression analysis and expressed as correlation coefficient
(R). Total number of peripheral blood leukocytes, albumin coefficient, sICAM-1, sVCAM-1 and sE-Selectin concentrations in plasma and CSF correlated significantly ( $p<0.001)$ with the infarct volume, the degree of neurological and functional deficit (Figs 1-5).

\section{Discussion}

Ischemic stroke is characterized by the disruption of cerebral blood flow. This reduction of cerebral blood flow results in energy failure and secondary biochemical disturbances, eliciting a robust in situ inflammatory response. Post-ischemic inflammation is a dynamic process involving a complicated set of interactions among various inflammatory cells and molecules. The resident inflammatory brain cells, microglia, are especially activated in response to ischemic insults, many of which are regulated by nuclear transcription factor, kappa B [8,41]. As a result, several inflammato- 
A

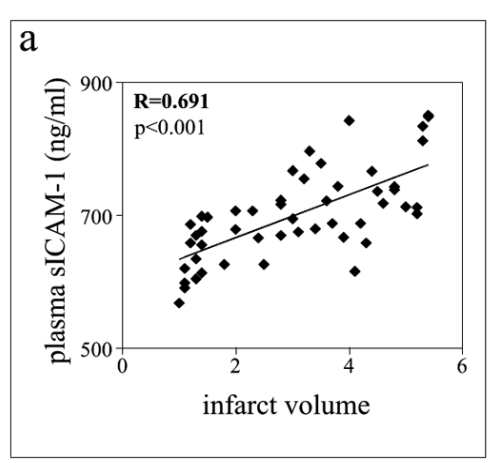

B

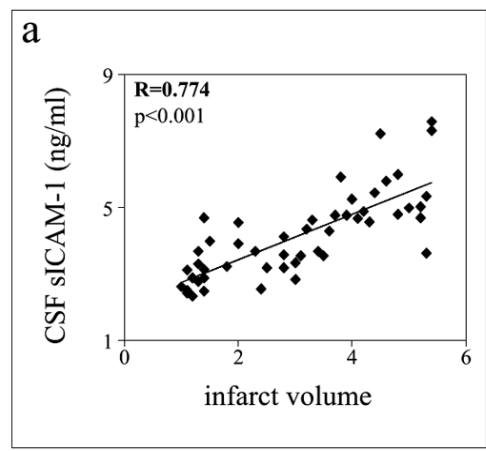

$\mathrm{b}$

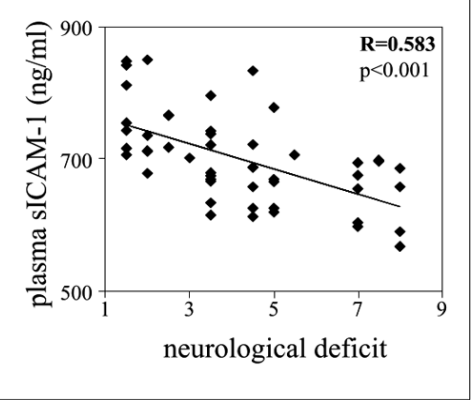

b

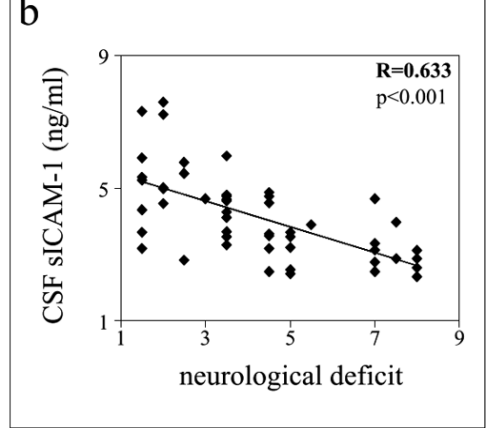

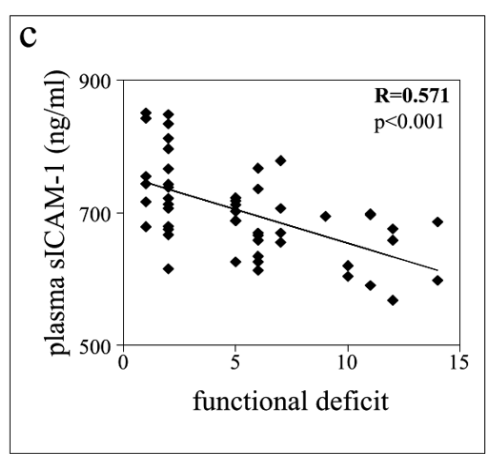

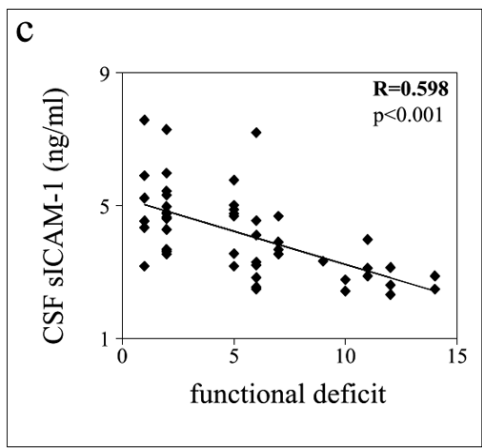

Fig. 3. Correlation between sICAM-1 concentration in A - plasma and B - CSF with: a - the infarct volume, using CT scan (cm); b-the degree of neurological deficit, using Canadian scale (1.5-10); $\mathrm{c}-$ the degree of functional deficit, using Barthel index (0-20). R - correlation coefficient; $p<0.001-$ represents statistical significance.

ry genes are expressed, leading to local generation of various cytokines, which in turn promulgate inflammatory signals. This can trigger immune responses ultimately leading to inflammatory cell activation and infiltration. Inside of inflammatory infiltrate the presence of macrophages, endothelial cells, leukocytes, microglia, oligodendrocytes, astrocytes and immune cells was found [21]. Meanwhile, endothelial cells lining the local cerebral blood vessels are stimulated to produce adhesion molecules, causing the migration of peripheral circulating leukocytes into the compromised brain tissue, an event that amplifies inflammatory signaling cascades [4,29]. Post-ischemic inflammation appears to serve multiple purposes, depending on its timing and magnitude, as well as the topographic distribution of various inflammatory molecules. Data from experimental manipulations of some inflammatory molecules are yielding insight into therapeutic strategies for ischemic stroke [41]. In the last few years, many studies focused on the inflammatory reactions in the zone of ischemic penumbra. The inflammation appeared as secondary phenomenon, as an answer to the acute injury of cerebral tissue [5,37].
Experimentally and clinically, stroke is followed by both acute and prolonged inflammatory responses characterized by the production of inflammatory cytokines and leukocyte infiltration into the brain. Our results showed significant increase in number of peripheral blood leukocytes with the highest value within the third and the fourth day after the insult. Also, we found significant correlation between number of leukocytes and the infarct volume, the neurological and the functional deficit. At the same time, we detected maximal increase of albumin coefficient, a marker of cerebrovascular barrier injury. Damage of the blood-brain barrier is a critical event leading to vasogenic edema, leukocytes invasion, and secondary brain injury. Blood-brain barrier damage can further potentiate brain tissue injury and contribute to secondary ischemic brain damage by permitting blood elements to enter the brain [35,39]. In acute brain infarction, generation of thrombin, oxygen radicals, and other stimulants of endothelial cells and leukocytes leads to an increase of both endothelial and leukocyte adhesion molecules expression, which in turn leads to further adhesion of leukocytes and subsequent release of cytokines and oxygen radicals, increase of 
A

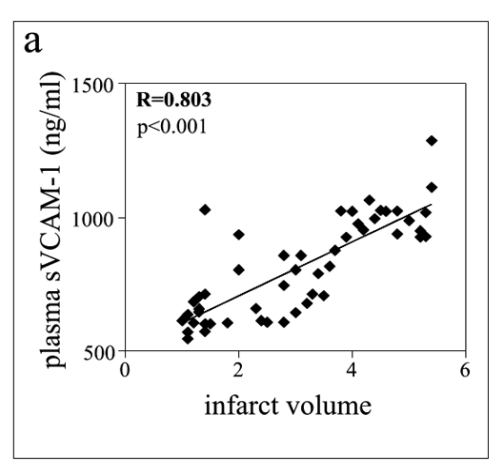

B

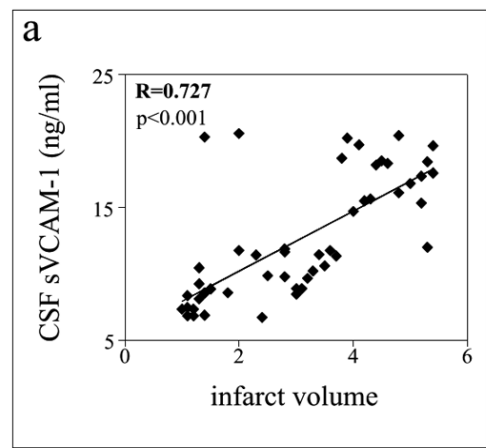

$\mathrm{b}$

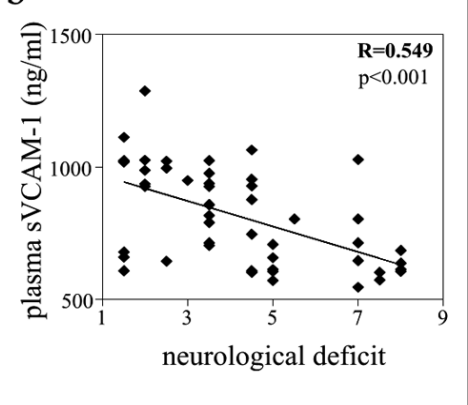

b

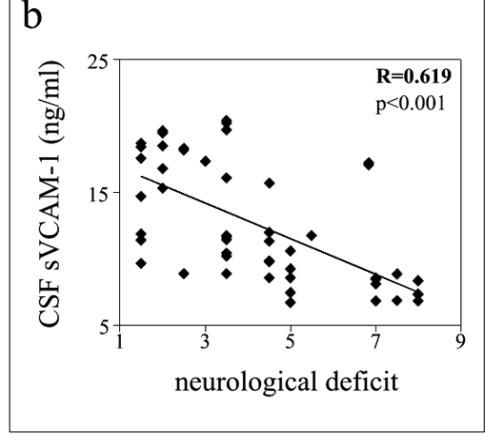

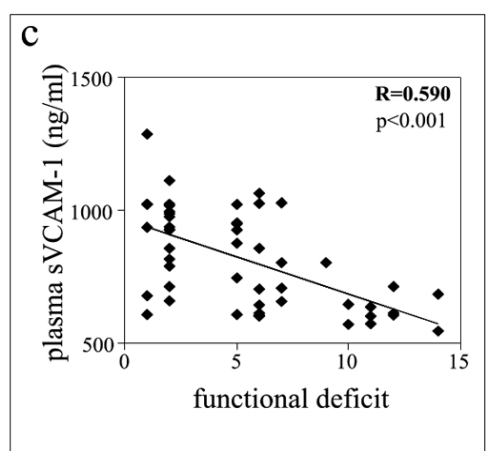

c

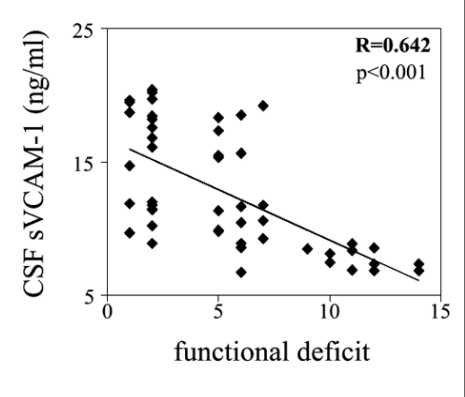

Fig. 4. Correlation between sVCAM-1 concentration in A - plasma and B - CSF with: a - the infarct volume, using CT scan (cm); b - the degree of neurological deficit, using Canadian scale (1.5-10); $\mathrm{c}-$ the degree of functional deficit, using Barthel index (0-20). R - correlation coefficient; $p<0.001-$ represents statistical significance.

tissue damage, and stimulation of endothelial cells and leukocytes, in an ongoing cycle $[12,33,41]$. There are not enough data about extra- and intracellular processes controlling the induction and the expression of adhesion molecules during the brain ischemia. Possible mediator could be nuclear factor $\kappa \mathrm{B}(\mathrm{NF}-\kappa \mathrm{B})$, which has important role in regulation of adhesion molecules transcription, activated by different factors induced by ischemia, including active oxygen forms, and proinflammatory mediator products, such as IL- 1 , TNF- $\alpha$ etc. $[6,21,26,38]$.

Until now, only few studies about soluble adhesion molecules in patients with brain ischemia were done, but data were controversial, due to small patient groups or inadequate controls $[2,9,14,15,32]$. Also, the results were explained in association with vascular CNS diseases. Our results showed significant increase of sICAM-1, sVCAM-1 and sE-Selectin concentrations in CSF and plasma in the acute period of brain infarction patients. Within the first seven days after the ischemia onset, temporal pattern non-linearity of sICAM-1, sVCAM-1 and sE-Selectin levels in plasma and CSF was detected. Maximal values were de- termined within the third and the fourth day after the insult. High levels of soluble adhesion molecules in CSF in the patients with BI are likely consequence of massive blood-brain barrier damage, which allows adhesion molecules to pass from plasma intro the CSF. However, in the patients with moderate and mild BI, neuronal damage is less expressed so levels of adhesion molecules are proportionally lower than in severe BI.

The role of each soluble adhesion molecule in pathophysiological events during brain ischemia is still unknown. It was previously shown that sICAM-1 in circulation can block lymphocyte adhesion on cerebral endothelia [31]. It is possible that sICAM have a role in the restriction of the other inflammatory reactions of endothelia. Also, sE-Selectin in blood acts as proinflammatory agents activating neutrophiles. But on the other side, sE-Selectin have surface competition ability and can inhibit leukocytes adhesion on endothelia in the inflammation site $[10,28]$. According to that, and our study as well, it is possible that soluble adhesion molecules can emerge pro-inflammatory and antiinflammatory characteristics, depending of the concen- 
A

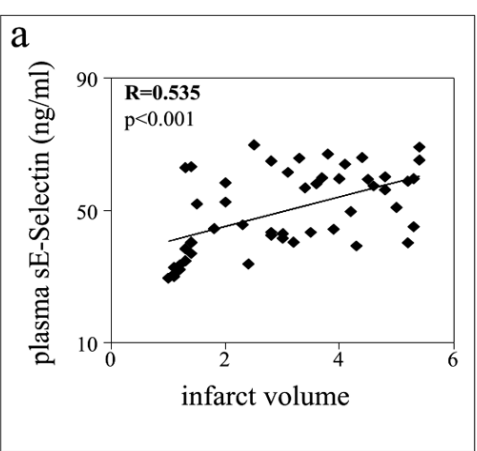

B

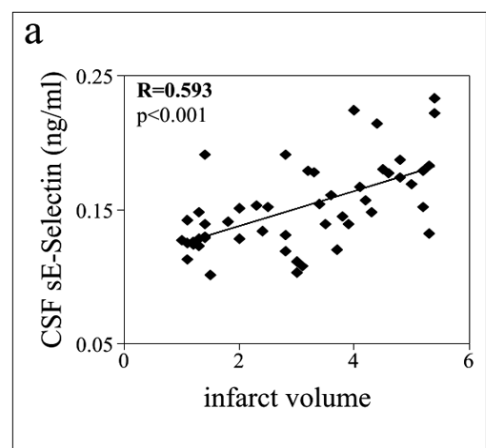

b

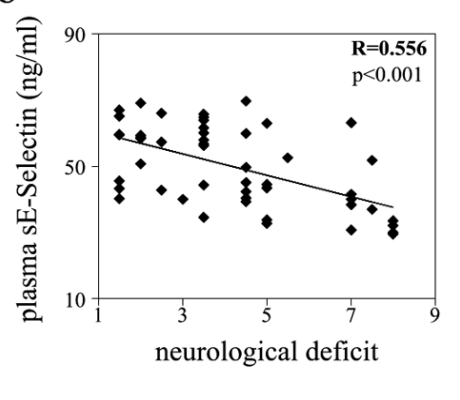

$\mathrm{b}$

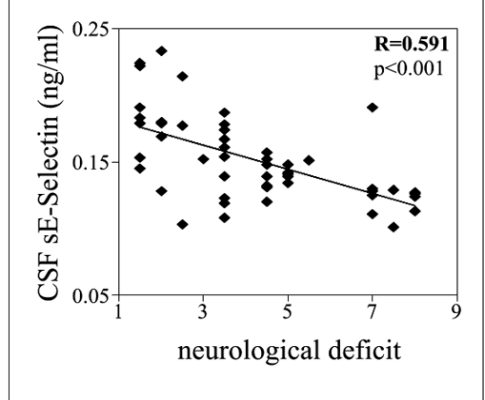

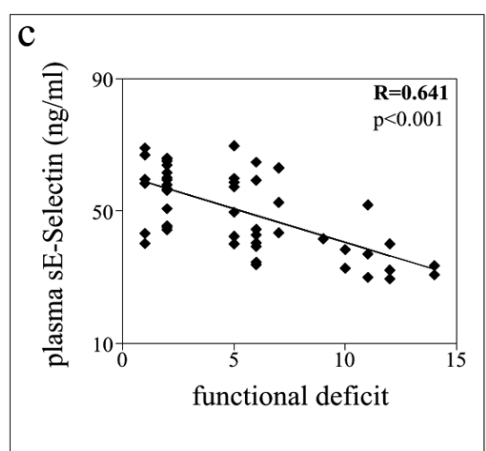

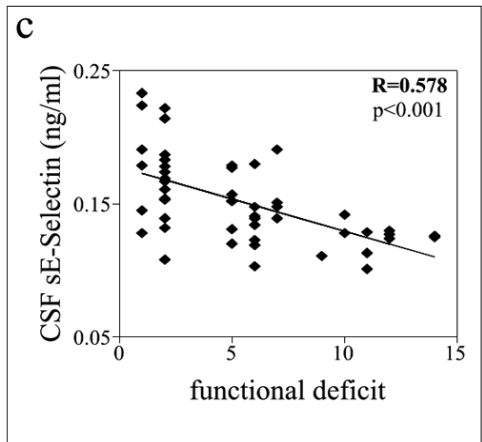

Fig. 5. Correlation between sE-Selectin concentration in A - plasma and B - CSF with: a - the infarct volume, using CT scan (cm); $\mathrm{b}-$ the degree of neurological deficit, using Canadian scale (1.5-10); $\mathrm{c}-$ the degree of functional deficit, using Barthel index (0-20). R - correlation coefficient; $p<0.001$ - represents statistical significance.

tration and presence of other mediators on a place of inflammation.

When ischemic neuron damage reached certain threshold toxical mediators are released. The inflammation, secondary damaged glia and vascular tissue can emphasized already existing neuron damage and spread it. There is certain time latency between these two events. This is the possibility for appropriate therapy use which will prevent the beginning and the expansion of secondary damage. Molecular markers of inflammation can be useful tools for the management of patients with ischemic stroke, during the acute phase and to predict prognosis and prevent the risk of a new vascular event.

\section{Conclusions}

In our study the highest values of investigated parameters were noted within the third and the fourth day after the brain ischemia onset, which is the suggested period of maximal intensity of inflammatory reactions. Also, we demonstrate significant correlation between determined parameters in CSF and plasma with the extent of infarct volume and the degree of neurological and the functional deficit. Our result showed that investigated parameters in CSF and blood represent a dynamic index of inflammatory events as one of the fundametal mechanisms responsible for neuron damage during acute phase of brain infarction.

\section{Acknowledgment}

This study was supported by grant of MMA (Grant No./06-08/B.6) and Ministry of Science and Technological Development Republic of Serbia (Grant No.143054).

\section{References}

[1] S.E. Akopov, N.A. Simonian and G.S. Grigorian, Dynamics of polymorphonuclear leukocyte accumulation in acute cerebral infarction and their correlation with brain tissue damage, Stroke 27 (1996), 1739-1743. 
[2] A. Bitsch, W. Klene, L. Murtada, H. Prange and P. Rieckmann, A longitudinal prospective study of soluble adhesion molecules in acute stroke, Stroke 29 (1998), 2129-2135.

[3] T.M. Carlos and J.M. Harlan, Leukocyte - endothelial adhesion molecules, Blood 84 (1994), 2068-2101.

[4] J. Castillo and R. Leira, Predictors of deteriorating cerebral infarct: role of inflammatory mechanisms. Would its early treatment be useful? Cerebrovascular Diseases 11 (2001), 40-48.

[5] A. Chamorro and J. Hallenbeck, The harms and benefits of inflammatory and immune responses in vascular disease, Stroke 37 (2006), 291-293.

[6] J. Clemens, D. Stephenson, B. Smalstig, E. Dixon and S. Little, Global ischemia activates nuclear factor-kB in forebrain neurons of rats, Stroke 28 (1997), 1073-1081.

[7] R. Cote, V.C. Hachinski, B.L. Shurvell, J.W. Norris and C. Wolfson, The Canadian Neurological Scale: A preliminary study in acute stroke, Stroke 17 (1986), 731-737.

[8] C.A. Davies, S.A. Loddick, R.P. Stroemer, J. Hunt and N.J. Rothwell, An integrated analysis of the progression of cell responses induced by permanent focal middle cerebral artery occlusion in the rat, Experimental Neurology 154 (1998), 199212.

[9] R. De Caterina, G. Basta, G. Lazzerini, G. Dell'Omo, R. Petrucci, M. Morale, F. Carmassi and R. Pedrinelli, Soluble vascular cell adhesion molecule-1 as a biohumoral correlate of atherosclerosis, Arteriosclerosis, Thrombosis, and Vascular Biology 17 (1997), 2646-2654.

[10] H.M. DeLisser, M. Christofidou-Solomidou, J. Sun, M.T. Nakada and K.E. Sullivan, Loss of endothelial surfaces expression of E-selectin in a patient with recurrent infections, Blood 94 (1999), 884-894.

[11] G. del Zoppo, I. Ginis, J.M. Hallenbeck, C. Iadecola, X. Wang and G.Z. Feuerstein, Inflammation and stroke: putative role for cytokines, adhesion molecules an iNOS in brain response to ischemia, Brain Pathology 10 (2000), 95-112.

[12] G. del Zoppo, Microvascular responses to cerebral ischemia/inflammation, Annals of the New York Academy of Sciences 823 (1997), 132-147.

[13] G. Feurestein, T. Liu and F. Barone, Cytokines, inflammation, and brain injury: role of tumor necrosis factor- $\alpha$, Cerebrovascular and Brain Metabolism Reviews 6 (1994), 341-360.

[14] C. Frijns, L. Kappelle, J. van Gijn, H. Nieuwenhuis, J. Sixma and R. Fijnheer, Soluble adhesion molecules reflect endothelial cell activation in ischemic stroke and in carotid atherosclerosis, Stroke 28 (1997), 2214-2218.

[15] C.J. Frijns and L.J. Kappelle, Inflammatory cell adhesion molecules in ischemic cerebrovascular disease, Stroke 33 (2002), 2115-2122.

[16] J. Garcia, K. Liu, Z. Ye and J. Gutierrez, Incomplete infarct and delayed neuronal death after transient middle cerebral artery occlusion in rats, Stroke 28 (1997), 2303-2310.

[17] J. Garcia, K. Liu, Y. Yoshida, J. Lian, S. Chen and G. del Zoppo, Influx of leukocytes and platelets in an evolving brain infarct (Wistar rats), American Journal of Pathology 144 (1994), 188-199.

[18] J.C. Grotta, N.A. Lemak, H. Gary, W.S. Fields and D. Vital, Does platelet antiaggregant therapy lessen the severity of stroke? Neurology 35 (1985), 632-636.

[19] H.P. Haring, E.L. Berg, N. Tsurushita, M. Tagaya and G. del Zoppo, E-selectin appears in nonischemic tissue during experimental focal cerebral ischemia, Stroke 27 (1996), 13861392.
[20] J. Harlan, Leukocyte - endothelial interactions, Blood 65 (1985), 513-525.

[21] J. Huang, U.M. Upadhyay and R.J. Tamargo, Inflammation in stroke and focal cerebral ischemia, Surgical Neurology 66 (2006), 232-245

[22] C. Iadecola and M. Alexander, Cerebral ischemia and inflammation, Current Opinion in Neurology 14 (2001), 89-94.

[23] A. Kaufmann, A. Firlik, M. Fukui, L. Wechsler, C. Jungries and $\mathrm{H}$. Yonas, Ischemic core and penumbra in human stroke, Stroke 30 (1999), 93-99.

[24] J. Kim, Cytokines and adhesion molecules in stroke and related diseases, Journal of the Neurological Sciences 137 (1996), 69-78.

[25] K. Kogure, Y. Yamasaki, Y. Matsuo, H. Kato and H. Onodera, Inflammation of the brain after ischemia, Acta Neurochirurgica 66 (1996), 40-43.

[26] C. Li, W. Browder and R.L. Kao, Early activation of transcription factor NF-kB during ischemia in perfused rat heart, American Journal of Physiology 276 (1999), H543-552.

[27] D. Male, J. Rahman, G. Pryce, T. Tamatani and M Miyasaka, Lymphocyte migration intro the CNS modeled in vitro: roles of LFA-1, ICAM-1 and VLA-4, Immunology 81 (1994), 366372.

[28] E. Morikawa, S. Zhang, Y. Seko, T. Toyoda and T. Kirino, Treatment of focal cerebral ischemia with synthetic oligopeptide corresponding to lectin domain of selectin, Stroke 27 (1996), 951-956.

[29] P. Nencini, C. Sarti, R. Innocenti, G. Pracucci and D. Inzitari, Acute inflammatory events and ischemic stroke subtypes, Cerebrovascular Diseases 15 (2003), 215-221.

[30] L. Pantoni, C. Sarti and D. Inzitari, Cytokines and cell adhesion molecules in cerebral ischemia: Experimental bases and therapeutic perspectives, Arteriosclerosis, Thrombosis, and Vascular Biology 18 (1998), 503-513.

[31] R. Polin, M. Bavbek, M. Shaffrey, K. Billups, C. Bogaev, N. Kassell and K. Lee, Detection of soluble E-selectin, ICAM-1, VCAM-1 and L-selectin in the cerebrospinal fluid of patients after subarachnoid hemorrhage, Journal of Neurosurgery 89 (1998), 559-567.

[32] C.J.S. Price, E.A. Warburton and D.K. Menon, Human cellular inflammation in the pathology of acute cerebral ischemia, Journal of Neurology Neurosurgery \& Psychiatry 74 (2003), 1476-1484.

[33] L. Ritter, J. Orozco, B. Coull and P. McDonagh, Leukocyte accumulation and hemodynamic changes in the cerebral microcirculation during early reperfusion after stroke, Stroke $\mathbf{3 1}$ (2000), 1153-1161.

[34] M. Rodríguez-Yáñez and J. Castillo, Role of inflammatory markers in brain ischemia, Current Opinion in Neurology 21 (2008), 353-357.

[35] B.K. Siesjo and P. Siesjo, Mechanisms of secondary brain injury, European Journal of Anesthesiology 13 (1996), 247268.

[36] D.T. Wade and RL. Mewer, Functional abilities after stroke: measurement, natural history and prognosis, Journal of $\mathrm{Neu}$ rology Neurosurgery \& Psychiatry 50 (1987), 177-182.

[37] Q. Wang, X.N. Tang and M.A. Yenari, The inflammatory response in stroke, Journal of Neuroimmunology 184 (2007), 53-68.

[38] C.H. Wong and P.J. Crack, Modulation of neuro-inflammation and vascular response by oxidative stress following cerebral ischemia-reperfusion injury, Current Medicinal Chemistry 15 (2008), 1-14. 
[39] M.A. Yenari, L. Xu, X.N. Tang, Y. Qiao and R.G. Giffard, Microglia potentiate damage to blood-brain barrier constituents: inprovement by minocycline in vivo and in vitro, Stroke $\mathbf{3 7}$ (2006), 1087-1093.

[40] R.L. Zhang, Z.G. Zhang and M. Chopp, Thrombolysis with tissue plasminogen activator alters adhesion molecule expression in the ischemic rat brain, Stroke 30 (1999), 624-629.

[41] Z. Zheng and M.A. Yenari, Post-ischemic inflammation: molecular mechanisms and therapeutic implications, Neurological Research 26 (2004), 884-892. 


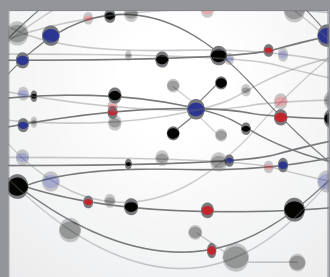

The Scientific World Journal
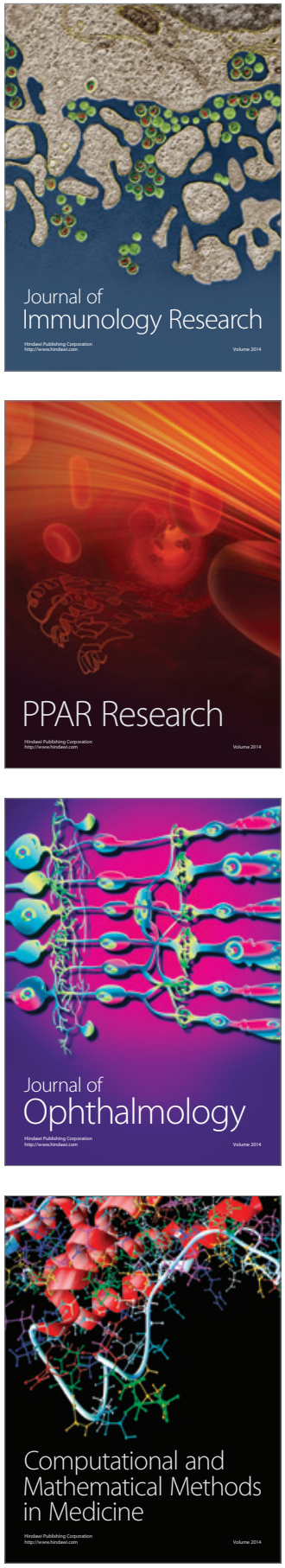

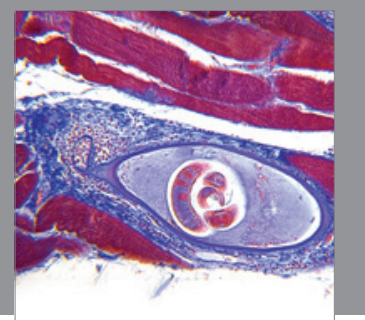

Gastroenterology

Research and Practice
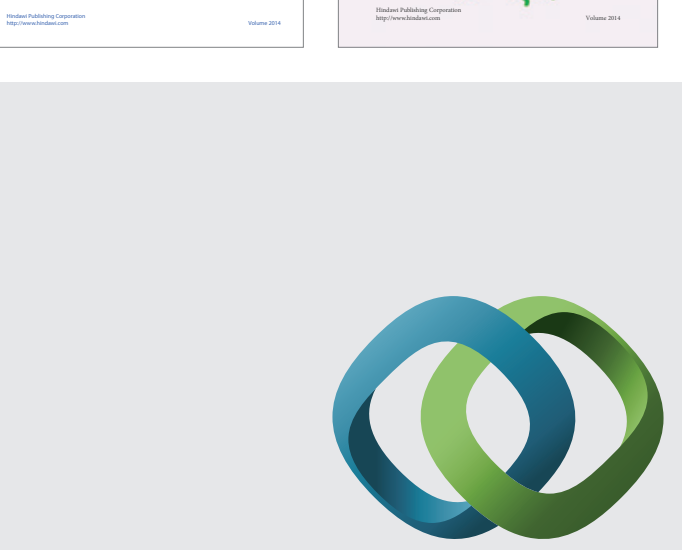

\section{Hindawi}

Submit your manuscripts at

http://www.hindawi.com
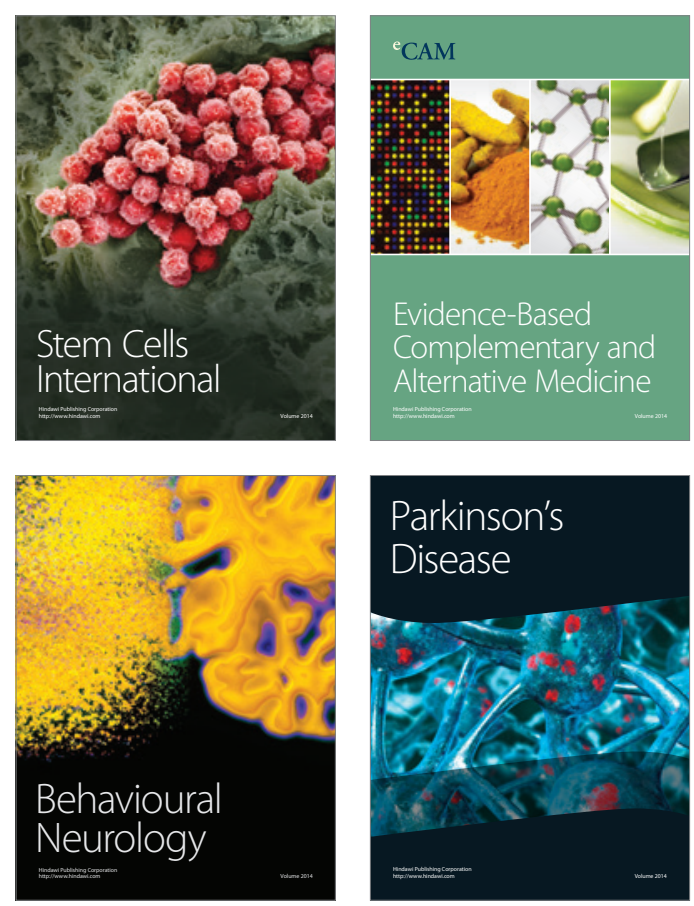

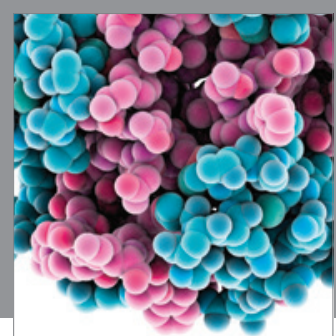

Journal of
Diabetes Research

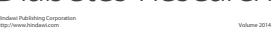

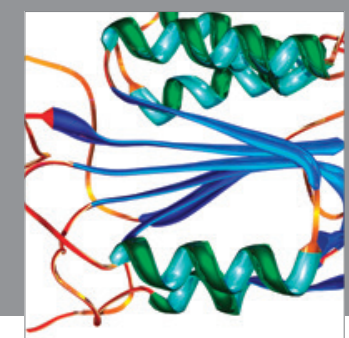

Disease Markers
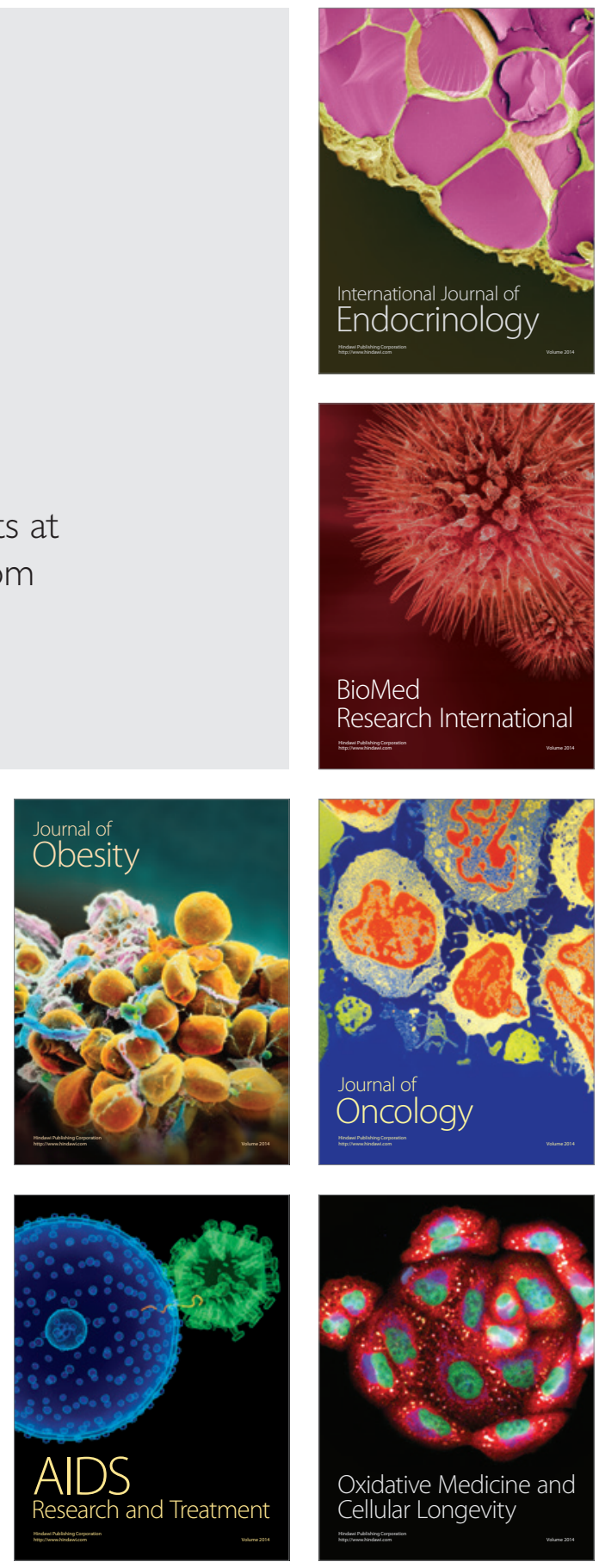